\title{
An X-Ray Diffraction Study of the Hexanuclear Complex of Bi(III) in Aqueous Perchlorate Solution. Determination of the
} Oxygen Positions

\author{
BENGT SUNDVALL
}

Department of Inorganic Chemistry, Royal Institute of Technology, S-100 44 Stockholm, Sweden

The X-ray scattering from a solution of basic bismuth(III) perchlorate, $4.68 \mathrm{M}$ in Bi(III), has been investigated in order to determine the oxygen positions in the hexanuclear complex. The data are shown to be consistent with a $\mathrm{Bi}_{6} \mathrm{O}_{4}(\mathrm{HO})_{4}^{6+}$ unit similar to that found in the crystal structures of $\mathrm{Bi}_{6} \mathrm{O}_{4}(\mathrm{HO})_{4}\left(\mathrm{ClO}_{4}\right)_{6} \cdot 7 \mathrm{H}_{2} \mathrm{O}$ and $\mathrm{Bi}_{6} \mathrm{O}_{4}(\mathrm{HO})_{4}$ $\left(\mathrm{NO}_{3}\right)_{6} \cdot \mathrm{H}_{2} \mathrm{O}$. The least squares refined value of the short $\mathrm{Bi}-\mathrm{Bi}$ distance in an assumed regular octahedral arrangement of $\mathrm{Bi}$ is $3.69_{7} \AA$. The oxygen atoms are situated above the octahedral faces of the $\mathrm{Bi}_{6}$ octahedron and each type of oxygen is tetrahedrally arranged around the centre of the complex. The $\mathrm{Bi}-\mathrm{O}^{2-}$ and $\mathrm{Bi}-\mathrm{HO}^{-}$bond lengths, determined from the positions of the corresponding peaks in the radial distribution curve, are 2.19 and $2.37 \AA$, respectively. A simple model for the coordination of oxygen atoms to $\mathrm{Bi}$ outside the complex is suggested.

The hydrolysis of bismuth(III) has been extensively studied and the predominance of a hexanuclear species in acidic solutions is well-established. ${ }^{1}$ From the results of emf measurements, Olin ${ }^{2}$ suggested the formula $\mathrm{Bi}_{6}(\mathrm{HO})_{12}^{6+}$ for the complex. It was, however, pointed out that this method cannot be used to decide on the number of molecules of solvation in the species and that the composition of the complex should therefore be written more generally as $\mathrm{Bi}_{6} \mathrm{O}_{n}(\mathrm{HO})_{12-2 n}^{6+}$. The possibility of the alternative formula $\mathrm{Bi}_{6} \mathrm{O}_{4}(\mathrm{HO})_{4}^{6+}$ was discussed. $^{3}$

In an X-ray solution study, Levy et al. ${ }^{4}$ found conclusive evidence for an octahedral arrangement of $\mathrm{Bi}$ in the hexanuclear complex. The oxygen atoms were suggested to be parts of the $\mathrm{HO}^{-}$ groups bridging two neighbouring $\mathrm{Bi}$ atoms, i.e. along an octahedral edge, corresponding to the formula $\mathrm{Bi}_{6}(\mathrm{HO})_{12}^{6+}$ (Fig. 1a).

This geometry was used by Maroni and Spiro ${ }^{5}$ in an interpretation of Raman and infrared measurements on crystals and solutions of basic perchlorate. They concluded that the vibrational data were consistent with the suggested arrangement.

Recently a hexanuclear complex $\mathrm{Bi}_{6} \mathrm{O}_{4}(\mathrm{HO})_{4}^{6+}$, still with an octahedral arrangement of $\mathrm{Bi}$, but with the oxygen atoms situated above the octahedral faces, was found as discrete units in a basic bismuth(III) perchlorate, $\mathrm{Bi}_{6} \mathrm{O}_{4}(\mathrm{HO})_{4}\left(\mathrm{ClO}_{4}\right)_{6}$ $7 \mathrm{H}_{2} \mathrm{O}$, ${ }^{6}$ and in the basic nitrates $\mathrm{Bi}_{6} \mathrm{O}_{4}(\mathrm{HO})_{4}$ $\left(\mathrm{NO}_{3}\right)_{6} \cdot \mathrm{H}_{2} \mathrm{O}^{7,8}$ and $\mathrm{Bi}_{6} \mathrm{O}_{4}(\mathrm{HO})_{4}\left(\mathrm{NO}_{3}\right)_{6} \cdot 4 \mathrm{H}_{2} \mathrm{O} .^{9}$ In this complex there is a significant difference between the $\mathrm{Bi}-\mathrm{O}^{2-}$ and the $\mathrm{Bi}-\mathrm{HO}^{-}$bond distances, and each type of oxygen forms an approximately tetrahedral shell surrounding the $\mathbf{B i}_{6}$ octahedron (Fig. 1b). In the paper on the basic perchlorate $^{6}$ it was shown that the ideal $T_{d}$ symmetry of this complex affords a better explanation of the vibrational spectra than the $O_{h}$ symmetry of the $\mathrm{Bi}_{6}(\mathrm{HO})_{12}^{6+}$ model.

Although it seems unlikely, the oxygen arrangement in the dissolved hexamer unit might differ from that in the crystals. Accurate X-ray diffraction measurements on solutions containing the hexamer could be expected to give information on the positions of the oxygen atoms in the dissolved complexes even if the contributions from the $\mathrm{Bi}-\mathrm{O}$ interactions to the scattering data are considerably smaller than those of the $\mathrm{Bi}-\mathrm{Bi}$ interactions. In order to find evidence for one or the other of the two models suggested, the present investigation was undertaken. 


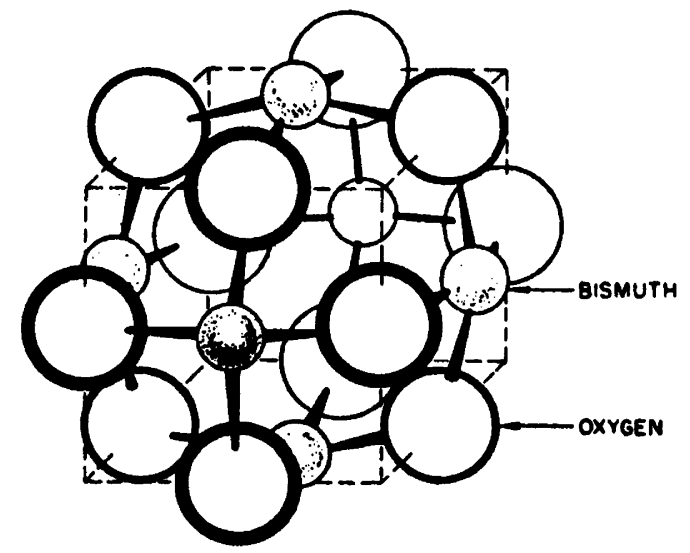

a

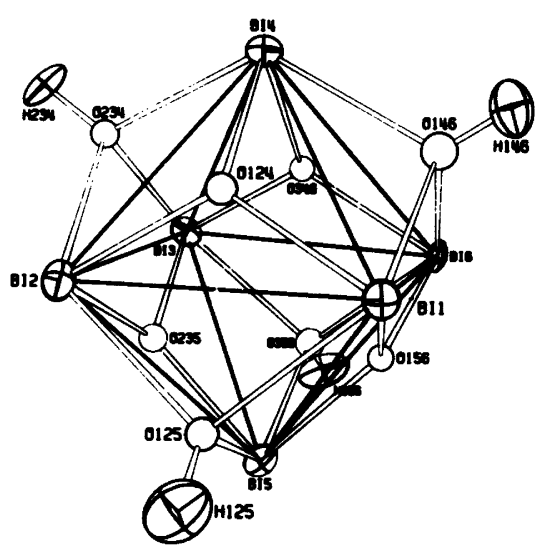

b

Fig. 1. a, Arrangement of bismuth and oxygen atoms in the model assumed by Levy et al. for the $\mathrm{Bi}_{6}(\mathrm{HO})_{12}^{6+}$ complex (from Ref. 4). $\mathrm{b}$, The complex $\mathrm{Bi}_{6} \mathrm{O}_{4}(\mathrm{HO})_{4}^{6+}$ found in the basic $\mathrm{Bi}(\mathrm{III})$ perchlorate $\mathrm{Bi}_{6} \mathrm{O}_{4}(\mathrm{HO})_{4}\left(\mathrm{ClO}_{4}\right)_{6} .7 \mathrm{H}_{2} \mathrm{O}$. Ellipsoids are scaled to include $50 \%$ probability. The $\mathrm{Bi}_{6}$ octahedron is marked out by thick single lines, not to be interpreted as indicating any $\mathrm{Bi}-\mathrm{Bi}$ bonding. Numbering of the atoms: see footnote in table $2 a$, Ref. 6.

\section{EXPERIMENTAL}

Solutions of basic perchlorate were prepared by dissolving purified $\mathrm{Bi}_{2} \mathrm{O}_{3}$ in $6 \mathrm{M} \mathrm{HClO}_{4}$. Only one solution, $4.68 \mathrm{M}$ in $\mathrm{BiOClO}_{4}$ and $0.63 \mathrm{M}_{\text {in }} \mathrm{HClO}_{4}$, was investigated. Using the equilibrium constants given by Olin ${ }^{2}$ and assuming that the activity factors do not change in going from $3 \mathrm{M} \mathrm{NaClO}_{4}$ medium to the solution investigated, it was estimated that the solution contained $97.2 \% \mathrm{Bi}$ (III) in the hexamer, $2.6 \%$ unhydrolyzed $\mathrm{Bi}^{3+}$ and $0.2 \%$ monomer $\mathrm{BiHO}^{2+}$.

The X-ray scattering measurements were carried out at $25 \pm 1{ }^{\circ} \mathrm{C}$ as previously described. ${ }^{10}$ The radiation used was $\operatorname{MoK} \alpha(\lambda=0.7107 \AA)$ and the scattered intensities were measured at discrete points in the $\theta$-range $1-69^{\circ}(2 \theta$ is the scattering angle) at intervals of $0.1^{\circ}$ for $1^{\circ}<\theta<21^{\circ}$ and $0.25^{\circ}$ for $21^{\circ}<\theta<69^{\circ}$. In general $10^{5}$ counts were accumulated for each point.

\section{DATA TREATMENT}

The data processing was performed according to the methods and formulas given in Ref. 11, by means of the computer programs KURVLR and PUTSLR. The intensities were corrected for polarization and normalized to a stoichiometric unit of volume containing one $\mathrm{Bi}$ atom, by comparison of the high-angle part of the experimental intensity curve with the calculated sum of the independent coherent and the incoherent scattering. Corrections for multiple scattering were made; they were in general less than $2 \%$. Reduced intensities $i_{\text {obs }}(s)$, where the angular variable is $s=4 \pi \sin \theta / \lambda$, were calculated, and the electronic radial distribution function $D(r)$ was evaluated. Peaks at distances below $1 \AA$ in the radial distribution function (RDF) were removed, thus eliminating the low-frequency components of the reduced intensity curve, not attributable to any reasonable intramolecular distances.

Scattering factors and corrections for the anomalous dispersion of $\mathrm{Bi}, \mathrm{Cl}$, and $\mathrm{O}$ were taken from International Tables. ${ }^{12}$ For $\mathrm{H}$ the scattering factors given by Stewart et al. ${ }^{13}$ were used and for molecular $\mathrm{H}_{2} \mathrm{O}$ those by Narten and Levy. ${ }^{14}$ The values for incoherent scattering were taken from Cromer and $\mathrm{Mann}^{15}$ for $\mathrm{Bi}$, from $\mathrm{Cromer}^{16}$ for $\mathrm{Cl}$ and $\mathrm{O}$, and from Compton and Allison ${ }^{17}$ for $\mathrm{H}$.

The modifying function used in the calculation of the RDF was $M(s)=\left\{\left[f_{\mathrm{Bi}}^{2}(0)+\left(\Delta f_{\mathrm{Bi}}^{\prime \prime}\right)^{2}\right] /\left[f_{\mathrm{Bi}}^{2}(s)+\right.\right.$ $\left.\left.\left(\Delta f_{\mathrm{Bi}}^{\prime \prime}\right)^{2}\right]\right\} \exp \left(-0.01 s^{2}\right)$ and the average scattering power $\rho_{0}$ was calculated as $\rho_{0}=\left[\left(\sum_{i} f_{i}\right)^{2}+\left(\sum_{i} \Delta f_{i}^{\prime \prime}\right)^{2}\right] /$ 


\section{Analysis of the experimentaldata}

Intensity data. Assuming a model for the solution, theoretical reduced intensities $i_{\text {calc }}(s)$ can be calculated. These may be fitted to the experimental intensities $i_{\text {obs }}(s)$ by adjusting the parameters defining the model in a least squares refinement procedure, thus minimizing the function $U=\sum w(s)$ $\left[i_{\text {obs }}(s)-i_{\text {calc }}(s)\right]^{2}$ where $w(s)$ is a weighting function. ${ }^{11}$ The refinement may be carried out for different $s$-intervals in order to obtain an estimate of the remaining systematic errors. The validity and limitations of this method of analyzing the experimental data have been previously thoroughly discussed. ${ }^{18}$

In spite of the fact that the predominance of a single species facilitated the interpretation of the experimental data in the present study, the testing of the full model by least squares refinement was obstructed by the much greater scattering power of $\mathrm{Bi}$ compared to the light atoms and by the complexity of the model.

The scattered intensities from the many intermolecular interactions in the solution are significant in the low-angle region, but become negligible at higher $s$-values due to high temperature factors. Hence the innermost part of the intensity curve was not used in the refinement of the intramolecular distances.

The contributions of the $\mathrm{Bi}-\mathrm{O}$ interactions also decrease with increasing $s$, compared to those of the $\mathrm{Bi}-\mathrm{Bi}$ interactions, due to the more rapid decrease of the scattering factor of oxygen. In the outer part of the intensity curve there were but small contributions from the $\mathrm{Bi}-\mathrm{O}$ interactions, the dominating part of the scattered intensities, by far, coming from the $\mathrm{Bi}-\mathrm{Bi}$ interactions. Thus, the refinement of a simplified model, comprising solely the $\mathrm{Bi}$ atoms, carried out in this s-interval, gave information on the $\mathrm{Bi}-\mathrm{Bi}$ distances in the complex which can be considered to be quite accurate.

All attempts to locate the oxygen atoms in the hexamer unit by use of the intensity curve were, however, unsuccessful due to the fact that the $s$ region, where the contributions of the $\mathrm{Bi}-\mathrm{O}$ interactions were appreciable, also contained considerable contributions from the intermolecular interactions.

Radial distribution function. Contributions to the radial distribution curve from the $\mathrm{Bi}-\mathrm{Bi}$ interactions in the complex were calculated with the use of the refined parameters and subtracted from the experimental RDF. The difference curve thus ob- tained showed peaks due to the remaining interactions in the solution (in fact the contributions from the perchlorate ions were subtracted as well in this step; the parameters for $\mathrm{ClO}_{4}^{-}$are well-known from earlier investigations in this laboratory). Some of the peaks could be attributed to $\mathrm{Bi}-\mathrm{O}$ interactions. The oxygen positions in the complex were then determined by adjusting the parameters of the $O$ atoms until a visible good fit was achieved between the experimental RDF and a distribution curve calculated on the basis of the model, in the appropriate region.

\section{RESULTS AND DISCUSSION}

The reduced experimental intensities multiplied with $s$ are shown in Fig. 4 (dots) and the radial distribution function $D(r)-4 \pi r^{2} \rho_{0}$ in Fig. 2a (solid

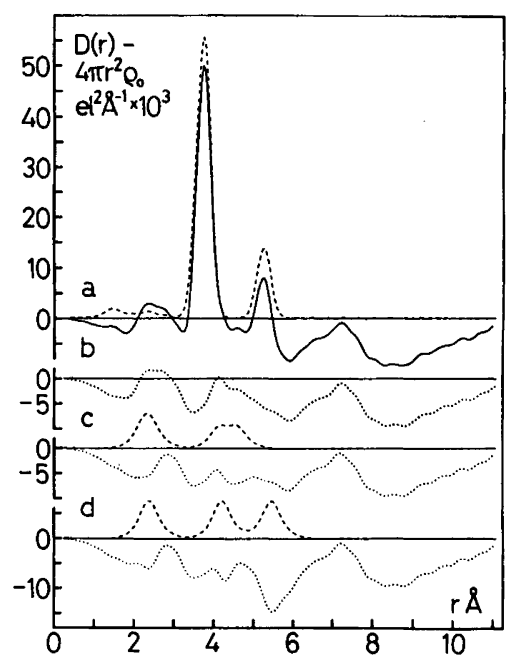

Fig. 2. a, The experimental distribution curve $D(r)-4 \pi r^{2} \rho_{0}$ (solid line) and the calculated peak shape for an idealized $\mathrm{Bi}_{6}$-octahedron including the contributions from $\mathrm{ClO}_{4}^{-}$(dashed line). $b$, The difference between the experimental and the calculated curve. The calculated peak shapes for the $\mathrm{Bi}-\mathrm{O}$ interactions in $\mathrm{c}, \mathrm{Bi}_{6} \mathrm{O}_{4}(\mathrm{HO})_{4}^{6+}$ and $\mathrm{d}$, $\mathrm{Bi}_{6}(\mathrm{HO})_{12}^{6+}$ (dashed lines), with the differences from the experimental curve of all the interactions in the respective models given below (dotted lines). The peaks at distances less than $1 \AA$ in the distribution curve, which were removed in the correction for lowfrequency components in the $i_{\text {obs }}(s)$ curve, were very small and cannot be visualized on the scale of this figure. 
line). As expected, the curves have essentially the same appearance as those of Levy et $\mathrm{al}^{4}$

The RDF shows two large, sharp peaks at about 3.7 and $5.2 \AA$, a small broad maximum at about at about $1.4 \AA$, two poorly resolved peaks at 2.4 and $2.8 \AA$, a small but clearly distinguishable peak at $4.6 \AA$ and a broad band of a number of superimposed maxima in the region of 6 to about $8 \AA$.

The small maximum at $1.4 \AA$ is probably due mostly to $\mathrm{Cl}-\mathrm{O}$ interactions in the perchlorate ions. In all calculations the contributions of $\mathrm{ClO}_{4}^{-}$were accounted for by assuming a tetrahedral geometry with a distance $r_{\mathrm{Cl}-\mathrm{O}}=1.40 \AA$ and with the temperature factors $b_{\mathrm{Cl}-\mathrm{O}}=0.001, b_{\mathrm{O}-\mathrm{o}}=0.002 \AA^{2}$.

As deduced by Levy et al., the two large maxima at 3.7 and $5.2 \AA$ are strongly indicative of an octahedral arrangement of the bismuth atoms, due to their relative size and the ratio of the distances being close to $\sqrt{2}$.

A least squares refinement of these two $B i-B i$ separations was performed for four different $s$ intervals and without any assumptions about the geometry, i.e. a refinement of two independent $\mathrm{Bi}-\mathrm{Bi}$ distances, the corresponding temperature factors and the frequencies of the interactions was performed.

The refined $\mathrm{Bi}-\mathrm{Bi}$ distances were, within the estimated limits of accuracy, constant throughout all the $s$-intervals. The average result was $3.70(1)$ and 5.23(2) $\AA$, where the standard deviations include an estimate of the systematic errors from the spread of the values in the different intervals. The result is in good agreement with the $\mathbf{B i}-\mathbf{B i}$ distances reported by Levy et al. (3.70 and $5.23 \AA$ ) and with the average $\mathrm{Bi}-\mathrm{Bi}$ distances in the basic perchlorate $^{5}$ (3.68 and $5.21 \AA$ from X-ray, 3.68 and $5.22 \AA$ from neutron diffraction) and nitrate ${ }^{6}(3.69$ and $5.21 \AA$ ).

The correlation between the temperature factors and the frequencies of distances was not severely large. The temperature factor of the short $\mathrm{Bi}-\mathrm{Bi}$ distance was fairly constant in the three outer $s$ intervals, the mean value being $0.0056(8) \AA^{2}$, while the temperature factor of the long $\mathrm{Bi}-\mathrm{Bi}$ distance varied between $0.0082(14)$ and $0.0016(23) \AA^{2}$, which indicates that the standard deviations (given in parentheses) obtained in the refinement are by far too low.

In the intermediate $s$-interval $5.0-16.5$, where the intramolecular contributions to the intensity curve are expected to dominate, while a reasonable number of experimental data are still included, the frequencies per $B i$ atom of the $3.70 \AA$ and the $5.22 \AA$ distances were $3.69(5)$ and $1.18(8)$, respectively (cf. 4 and 1 for an octahedron, which reduce to 3.89 and 0.97 if only $97.2 \%$ of the total $\mathrm{Bi}$ (III) is considered to be in the hexamer). The result is in agreement with the values found by Levy et al. by estimating the peak areas.

Since the octahedral arrangement of the bismuth atoms is very well-established, another refinement based on a regular octahedron of $\mathrm{Bi}$ was performed, thus reducing the number of parameters to one positional parameter and one temperature factor. The result corresponded to a $\mathrm{Bi}-\mathrm{Bi}$ separation of 3.697(1) $\AA, b_{\mathrm{Bi}-\mathrm{Bi}}=0.0056(8) \AA^{2}$ (s-interval $7.6-16.5$; the standard deviations are those calculated in the refinement process and do not include systematic errors).

The significance of the value of the temperature factor of the short $\mathrm{Bi}-\mathrm{Bi}$ interaction was checked by refining a temperature factor when fixed positional parameters from the crystal structure model were used (basic perchlorate, neutron data). The result, $b_{\mathrm{Bi}-\mathrm{Bi}}=0.0042(1) \AA^{2}$ (s-interval $7.6-16.5$ ), is in good agreement with the previous value. The distortions from an ideal octahedron in the crystal structure is expected to lead to a somewhat lower $b$-value.

Determination of the oxygen positions. The peak shape of the $\mathrm{RDF}$ for the idealized $\mathrm{Bi}_{6}$ octahedron is shown in Fig. 2a (dashed line; $\mathrm{ClO}_{4}^{-}$-interactions included). A difference curve calculated by subtracting these contributions from the $D(r)-4 \pi r^{2} \rho_{0}$ function is shown in Fig. $2 b$. In this curve there remain a partially unresolved double maximum at about 2.3 and $2.8 \AA$ and two small but distinct peaks at 4.2 and $4.6 \AA$.

These remaining peaks may now be compared with the theoretical $\mathrm{Bi}-\mathrm{O}$ interactions in the $\mathrm{Bi}_{6} \mathrm{O}_{4}(\mathrm{HO})_{4}^{6+}$ model, Fig. 2c, and with those in the $\mathrm{Bi}_{6}(\mathrm{HO})_{12}^{6+}$ model, Fig. $2 \mathrm{~d}$ (dashed lines). The difference curves (dotted lines) calculated by subtracting the contributions from all the interactions in the respective models from the experimental RDF are also given. The theoretical curve of the $\mathrm{Bi}_{6}(\mathrm{HO})_{12}^{6+}$ model was calculated with $r_{\mathrm{Bi}-\mathrm{o}}=2.33$ $\AA$ as reported by Levy et al., and for the $\mathrm{Bi}_{6} \mathrm{O}_{4}(\mathrm{HO})_{4}^{6+}$ model the values 2.19 and $2.37 \AA$ were taken for the $\mathrm{Bi}-\mathrm{O}^{2-}$ and $\mathrm{Bi}-\mathrm{HO}^{-}$distances, respectively. The average $\mathrm{Bi}-\mathrm{O}^{2-}$ and $\mathrm{Bi}-\mathrm{HO}^{-}$distances in the basic perchlorate crystals are 2.15 and $2.40 \AA$, respectively, but a slightly better fit to the experimental curve was achieved 
with the former values. For both models the refined $\mathrm{Bi}-\mathrm{Bi}$ distance in the assumed regular octahedral arrangement and its temperature factor were used and for the oxygen interactions the values $b_{\mathrm{Bi}-\mathrm{O}}=$ $0.0078, b_{0-0}=0.010 \AA^{2}$ were chosen.

The short $\mathrm{Bi}-\mathrm{O}$ interactions in $\mathrm{Bi}_{6}(\mathrm{HO})_{12}^{6+}$ will, of course, result in a single peak at $2.33 \AA$, and for $\mathrm{Bi}_{6} \mathrm{O}_{4}(\mathrm{HO})_{4}^{6+}$ the two different short $\mathrm{Bi}-\mathrm{O}$ separations give a single unresolved maximum at about the same distance. Accordingly the maximum at $2.3 \AA$ in the RDF can be explained equally well by both models.

The situation is, however, different for the long $\mathrm{Bi}-\mathrm{O}$ interactions. The $\mathrm{Bi}_{6} \mathrm{O}_{4}(\mathrm{HO})_{4}^{6+}$ model gives two peaks at 4.11 and $4.58 \AA$, which is in agreement with the difference curve $2 \mathrm{~b}$. $\mathrm{Bi}_{6}(\mathrm{HO})_{12}^{6+}$ gives peaks at 4.18 and $5.44 \AA$. The first of these could be assigned to the $4.2 \AA$ peak in the difference curve $2 b$, but for the second peak there is no corresponding experimental maximum. This distance would certainly be overlapped by the large peak due to the diagonal $\mathrm{Bi}-\mathrm{Bi}$ separation at $5.22 \AA$, but some residual effect would most probably have been exhibited in the difference curve, had the $\mathrm{Bi}_{6}(\mathrm{HO})_{12}^{6+}$ complex been the true model. This is confirmed by the difference curve in Fig. 2d, which shows a deep minimum at the actual distance. The difference curve for $\mathrm{Bi}_{6} \mathrm{O}_{4}(\mathrm{HO})_{4}^{6+}$ in Fig. $2 \mathrm{c}$ is, on the contrary, fairly smooth in this region. Hence the type of arrangement of oxygen atoms around the bismuth octahedron found in the crystal structures gives a better explanation of the experimental data than the model assumed by Levy et al.

Coordination to $\mathrm{Bi}$ by atoms outside the complex. Four oxygen atoms within the $\mathrm{Bi}_{6} \mathrm{O}_{4}(\mathrm{HO})_{4}^{6+}$ complex are coordinated on one side of each $\mathrm{Bi}$ in a trigonal bipyramidal geometry with the equatorial apex pointing outwards from the centre of the complex empty (where the lone $6 s^{2}$ electron pair of $\mathrm{Bi}(\mathrm{III})$ is supposed to be located ${ }^{6}$ ). The completion of the coordination polyhedron around $\mathrm{Bi}$ is, in the crystal structures, achieved by oxygen atoms from water molecules and perchlorate or nitrate groups. In the basic perchlorate there are four outer -oxygens at $\mathrm{Bi}-\mathrm{O}$ distances varying between 2.61(1) and $3.00 \AA$, average value $2.81 \AA$. It is reasonable to assume that in the solution the coordination to $\mathrm{Bi}$ outside the somplex occurs at about the same distances and results in the peak at $2.8 \AA$ in the distribution curve. Hydrogen bonding interactions between the solvent molecules and between solvent molecules and the $\mathrm{HO}^{-}$groups in the complex will also contribute to this peak. However, a calculation shows that these contributions account for only a minor part of the peak.

An attempt to account for this coordination to Bi by oxygen atoms outside the complex was made by introducing an extra oxygen atom with a multiplicity of 24 . The location of the atom was selected at a distance of $2.81 \AA$ from the coordinating $\mathrm{Bi}$ and at $4.08 \AA$ from the next nearest $\mathrm{Bi}$ in the complex, the latter $\mathrm{Bi}-\mathrm{O}$ interaction accounting for the residual peak at $4.1 \AA$ in the difference curve in Fig. 2c. This location is approximately in agreement with the average position of an outer coordinated oxygen in the basic perchlorate. The temperature factor is expected to increase with an increasing $\mathrm{Bi}-\mathrm{O}$ distance, and hence $b_{\mathrm{Bi}-\mathrm{o}}$ was chosen as $0.013 \AA^{2}$ for the shortest distance and successively increased to $0.053 \AA^{2}$ for the longest $\mathrm{Bi}-\mathrm{O}$ interactions.

The remaining diffuse interactions in the solution were approximated by an even electron distribution around the complexes outside radii approximately corresponding to the radii of the species.

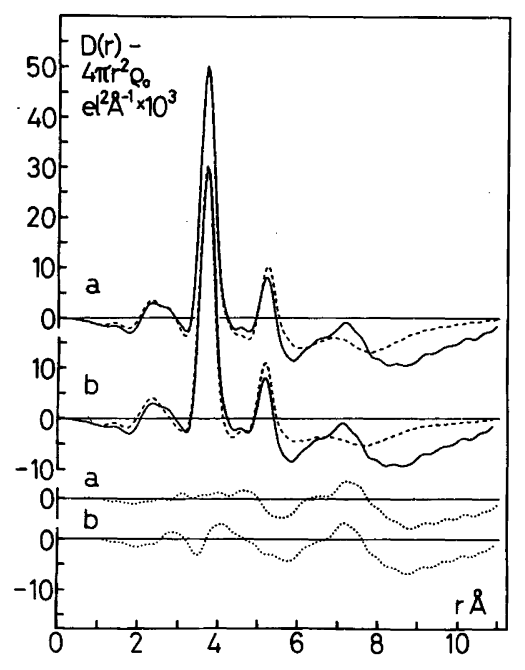

Fig. 3. The experimental distribution curve $D(r)-$ $4 \pi r^{2} \rho_{0}$ (solid lines). a, The calculated distribution curve for the final idealized model. $b$, The calculated distribution curve based on the crystal structure arrangement in the basic perchlorate (dashed lines). In both models the extra coordinated oxygen atoms and an uniform electron distribution outside the complexes are included. The corresponding difference curves are given at the bottom of the figure (dotted lines). 


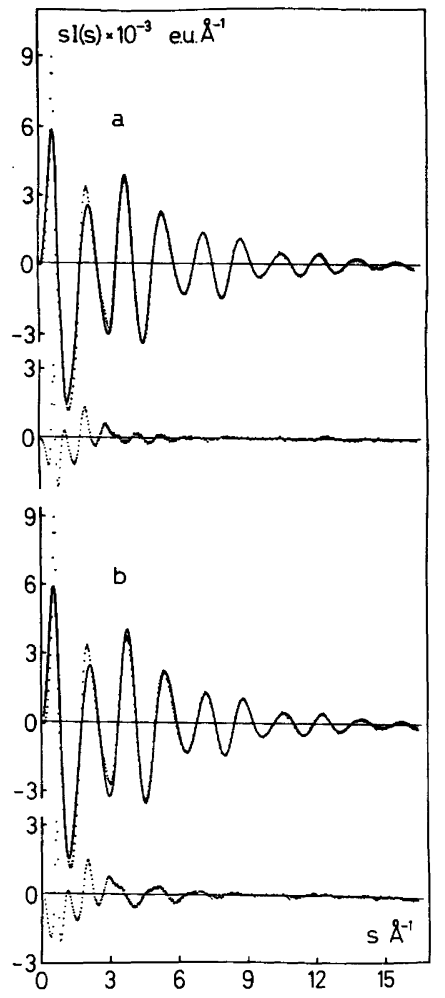

Fig. 4. The experimental reduced intensities si(s) obs (dotted lines) and the calculated intensity curves si $(s)_{\text {calc }}$ a, The final idealized model. b, Based on the crystal structure (solid lines), with the corresponding differences given below (dotted lines).

The resulting theoretical distribution curve in Fig. 3a (dashed line) and the theoretical intensity curve in Fig. 4a (solid line) show good agreement with the experimental data. It may be noted that the very long interactions between the $\mathrm{Bi}$ atoms in the complex and the extra oxygen outside the complex partly account for the overlapping peaks in the $6-8 \AA$ region in the RDF (Fig. 3a).

For comparison, the theoretical distribution curve (Fig. 3b) and the intensity curve (Fig. 4b) calculated from the crystal structure model of the basic perchlorate are given, including the outer coordinated oxygen atoms and an even electron distribution outside the complexes. These curves show essentially the same features as those calculated from the idealized model, except that the $2.8 \AA$ peak is less marked in the distribution curve for the crystal structure. This may be taken to indicate that the coordination of oxygen atoms to $\mathrm{Bi}$ outside the complex is more regular in the solution, which is quite reasonable. In the solution, solvent molecules are likely to coordinate, while the coordination in the solid is accomplished mainly by perchlorate groups, the positions of which are restricted by packing effects.

The conclusion that the complex found in the crystalline state is also present in solution, is further supported by the similarity of the Raman spectra of crystalline and dissolved basic bismuth perchlorate. ${ }^{5}$

Acknowledgements. I am greatly indebted to Drs. G. Johansson and M. Sandström for much helpful advice and interest in this work. I also thank Prof. I. Grenthe for valuable criticism of the manuscript and Mr. E. Hansen for technical assistance.

\section{REFERENCES}

1. Baes, C. F. and Mesmer, R. E. The Hydrolysis of Cations, Wiley, New York 1976.

2. Olin, A. Acta Chem. Scand. 11 (1957) 1445.

3. Olin, A. Sven. Kem. Tidskr. 73 (1961) 482.

4. Levy, H. A., Danford, M. D. and Agron, P. A. J. Chem. Phys. 31 (1959) 1458.

5. Maroni, V. A. and Spiro, T. G. J. Am. Chem. Soc. 88 (1966) 1410.

6. Sundvall, B. Inorg. Chem. To be published.

7. Sundvall, B. Acta Chem. Scand. A 33 (1979) 219.

8. Lazarini, F. Acta Crystallogr. B 35 (1979) 448.

9. Lazarini, F. Cryst. Struct. Commun. 8 (1979) 69.

10. Johansson, G. Acta Chem. Scand. 25 (1971) 2787; Ibid. 20 (1966) 553.

11. Johansson, G. and Sandström, M. Chem. Scr. 4 (1973) 195.

12. International Tables for $X$-Ray Crystallography, Kynoch Press, Birmingham 1974, Vol. IV.

13. Stewart, R. F., Davidson, E. R. and Simpson, W. T. J. Chem. Phys. 42 (1965) 3175.

14. Narten, A. H. and Levy, H. A. J. Chem. Phys. 55 (1971) 2263.

15. Cromer, D. T. and Mann, J. B. J. Chem. Phys. 47 (1967) 1892.

16. Cromer, D. T. J. Chem. Phys. 50 (1969) 4857.

17. Compton, A. H. and Allison, S. K. X-Rays in Theory and Experiment, Van Nostrand, New York 1935.

18. Johansson, G. and Sandström, M. Acta Chem. Scand. A 31 (1977) 132.

Received July 9, 1979. 